Eur Psychiatry Ed. Esp. (2002); 9: 351-356

EDITORIAL

\title{
¡Feliz cumpleaños, neurolépticos! Cincuenta años después: la locura de la duda
}

\author{
E. Stip \\ Universidad de Montreal, Hospital Louis-H. Lafontaine, Montreal, Canadá
}

\begin{abstract}
Resumen - Dado que celebramos el quicuagésimo cumpleaños de la introducción de los neurolépticos en psiquiatría, el autor propone echar un vistazo a algunos resultados relacionados con la práctica terapéutica. Después de una breve revisión cronológica de las publicaciones de las prácticas clínicas y los modelos teóricos que han controlado el tratamiento farmacológico de la esquizofrenia, el autor presenta una revisión crítica de cuatro meta-análisis. Desde el informe inicial de Delay, Deniker y Harl, la historia de los neurolépticos comprende varios períodos. En 1963 se propuso la teoría hiperdopaminérgica de la psicosis. Otro período comenzó con los modelos basados principalmente en la hipótesis del bloqueo relativo de los receptores de serotonina/dopamina. Más recientemente, un nuevo marco para comprender el efecto diferencial de los antipsicóticos se relaciona con la modulación apropiada (por ejemplo, la disociación rápida) del receptor $\mathrm{D}_{2}$ solo. El concepto de atipicidad se ha convertido en una nueva perspectiva para la investigación y para comercializar nuevos compuestos. Sin embargo, después de 50 años de fármacos neurolépticos, ¿podemos responder a las simples preguntas siguientes?: ¿Son los neurolépticos eficaces en el tratamiento de la esquizofrenia? ¿Hay diferencias entre los neurolépticos atípicos y los convencionales? ¿Cuál es la eficacia y la seguridad de los nuevos medicamentos antipsicóticos comparado con la eficacia y la seguridad de la clozapina? En realidad, las respuestas que los meta-análisis dan a estas simples preguntas deberían provocar en nosotros una gran humildad. Si deseamos basar la psiquiatría en la medicina basada en la evidencia, corremos un riesgo real al mirar más de cerca a lo que durante mucho tiempo se han considerado hechos. Cada psiquiatra debe continuar siendo crítico, escético y (no excesivamente) optimista y aprender para integrar los aspectos positivos de nuestra base creciente de conocimientos.
\end{abstract}

\section{antipsicóticos / neurolépticos / medicina científico-estadística / historia de los neurolépticos / clozapina / meta-análisis}

\section{INTRODUCCIÓN}

Se suponía que el primer ensayo que utilizó neurolépticos con fines psiquiátricos había tenido lugar el 9 de noviembre de 1951 en el hospital psiquiátrico Villejuif en París [6]. Consistió en el consumo de clorpromacina por un psiquiatra del personal del hospital para comprobar su efecto emocional potencial. En realidad, los medicamentos antipsicóticos los utilizó por primera vez en Francia en 1952 el equipo del Hospital Val-de-Grace (un hospital militar donde reciben tratamiento políticos famosos de la República) [22] y luego el equipo del Hospital de Santa Ana en París [23, 7-19, 21]. La clorpromacina (Rhone Poulenc), sintetizada y puesta a prueba por Charpentier y Courvoisier en 1950, se utilizaba para la llamada hibernación artificial en anestesia y cirugía por Henri Laborit [1, $26,32]$. Este cirujano naval advirtió el efecto tranqui-

Stip E. Happy birthday neuroloptics! 50 years later: la folie du doute. Eur Psychiatry 2002; 17: 115-9. 
lizante sin sedación que el fármaco inducía y predijo un interés para las enfermedades psiquiátricas. En el momento del descubrimiento de los neurolépticos, su mecanismo de acción era totalmente desconocido. En 1957 se identificó la dopamina como neurotransmisor central. Fue únicamente en 1963 cuando el bloqueo del receptor de la dopamina se reconoció como el efecto principal de los neurolépticos [4]. Incluso mientras el debate en torno a la hipótesis de la proporción 5HT2/D2 está todavía vivo [30, 31, 34], hemos advertido todos el renacimiento de la hipótesis de la dopamina [24], una hipótesis basada en el interesante componente diferencial k-off de la fijación, que postula que el efecto de un fármaco es proporcional a la velocidad cinética de comienzo (k-on) y de cancelación (k-off) de la fijación del fármaco al receptor. Contrariamente a las hipótesis multirreceptor, el predictor predominante de la atipicidad de los antipsicóticos es la disociación rápida del receptor $\mathrm{D} 2$, que está presente con claridad con la clozapina y la quetiapina. Dado que todos los antipsicóticos tienen una k-on bastante similar, sólo una diferencia en una disociación rápida del receptor $\mathrm{D} 2$ conduce al efecto antipsicótico atípico [25]. Sin embargo, al principio, Delay y Deniker desarrollaron progresivamente la noción de neurolépticos, dando una definición con cinco puntos: estado de indiferencia, acción antipsicótica, inducción de parkinsonismo, un efecto subcortical importante. Originalmente, típico significaba que inducía síntomas extrapiramidales. Después de la familia química de las fenotiacinas, siguieron butirofenonas y otras como las dibenzodiacepinas "en que la clozapina está a la cabeza". El término típico varía según los autores, la dosis y la comercialización [29, 33].

Dado que celebramos el quincuagésimo cumpleaños de la introducción de los neurolépticos en psiquiatría, ¿podríamos echar un vistazo a algunos resultados relacionados con la práctica terapéutica? La medicina basada en la evidencia (MBE), como indica la propia expresión, es una medicina que descansa en sólidas pruebas científicas. Desde hace ya algún tiempo, la MBE ha aumentado en importancia en varias áreas, incluida la pedagogía, donde está cambiando el modo de enseñar medicina y produciendo una racionalización de la buena práctica clínica. La psiquiatría ha abrazado esta tendencia y, con fines de autoexamen, recurre al meta-análisis, es decir, un procedimiento para procesar estadísticamente los estudios como datos. En otras palabras, es un método para revisar y evaluar las publicaciones de investigación.
En cierto sentido, el meta-análisis es análogo a la investigación experimental en su propósito de integrar y analizar estadísticamente los resultados. Su población de estudio consta de todos los trabajos publicados sobre un asunto dado. Sobre esta población se llevan a cabo análisis estadísticos para verificar diversos supuestos, que muy a menudo tienen que ver con preocupaciones de la evaluación del tratamiento. El meta-análisis se puede descomponer en seis pasos. En primer lugar, se debe formular una pregunta de investigación, por ejemplo: ¿Son más seguros y más eficaces los antipsicóticos atípicos que los neurolépticos convencionales? En segundo lugar, se debe emprender una revisión completa de las publicaciones pertinentes. En tercer lugar, se deben codificar todas las entradas de datos y variables (por ejemplo, el número de participantes, la duración del período doble ciego, la dosis de haloperidol). En cuarto lugar, se ha de establecer un índice de tamaño del efecto. En quinto lugar, se debe llevar a cabo un análisis estadístico de la distribución del tamaño del efecto. Por último, se han de interpretar los resultados. Después de 50 años de fármacos neurolépticos, ¿podemos responder a las preguntas simples siguientes?: ¿Son los neurolépticos eficaces en el tratamiento de la esquizofrenia? ¿Hay diferencias entre los neurolépticos atípicos y los convencionales? ¿Cuál es la eficacia y la seguridad de los nuevos fármacos antipsicóticos comparado con la eficacia y la seguridad de la clozapina?

¿Son eficaces los neurolépticos en el tratamiento de la esquizofrenia? Un meta-análisis reciente dirigido por Thornley y Adams [35] examinó el contenido y la calidad de 2.000 ensayos controlados realizados de 1948 a 1997. Los autores recabaron la mayor cantidad posible de datos buscando Biological Abstracts, CINAHL, la Biblioteca Cochrane, Embase, LILACS, Psychlit, PSYINDEX, Medline y Sociofile. En conjunto, esto representaba 30.000 informes electrónicos y 6.000 artículos. Luego codificaron la calidad de los estudios según diversos criterios, incluido el uso del método doble ciego, la distribución al azar y la duración. De acuerdo con esto, se consideró que sólo un $1 \%$ de los estudios era de buena calidad en una escala de 1 a 5 . Un tercio recibió una puntuación de 2, que indicaba deficiencias cualitativas. Los peores estudios se habían realizado en los Estados Unidos, pero los autores advirtieron una mejoría en la calidad con el tiempo. La puntuación media de calidad fue un mediocre 2,5. El número medio de participantes en los ensayos era 65 , y sólo un $1 \%$ de los estudios tenía poder estadístico suficiente. Únicamente un 3\% de los 
ensayos tenía un tamaño de muestra de 150 o más, que es necesario para demostrar una diferencia intergrupo del $20 \%$, y el $50 \%$ tenía menos de 50 participantes. La duración del $54 \%$ de los ensayos era inferior a 6 semanas; sólo un quinto de ellos duró 6 meses. Además, un $25 \%$ de los estudios no utilizó instrumento de medida para evaluar los cambios. En el resto de la muestra de estudios, se identificaron 640 instrumentos diferentes de medida. Thornley y Adams concluyeron: "Es probable que la calidad uniformemente mala de los informes haya resultado en una estimación en exceso optimista de los efectos del tratamiento." Los autores destacaron también que el número inusualmente grande de escalas de estimación utilizado y la duración limitada de los estudios puede derivar en hallazgos significativos equívocos. Durante 50 años, la clorpromacina se ha conocido por su nombre comercial francés Largactil®, que significa en francés "gran acción". ¿Deberíamos cambiarlo quizá a "Petitactil" o "Pequeñactil"?

¿Son los neurolépticos atípicos más eficaces que los convencionales? En 1999, Leucht y cols. [27] publicaron un meta-análisis sobre la eficacia y los efectos secundarios extrapiramidales de los nuevos antipsicóticos. Sus hallazgos mostraron que los nuevos fármacos son más eficaces que el placebo pero la magnitud del efecto es moderada. Cuando se estudiaron los síntomas negativos, los antipsicóticos nuevos eran más efectivos que el placebo; sin embargo, también lo era el neuroléptico convencional haloperidol. Además, aunque en las comparaciones directas algunos medicamentos atípicos mostraban una superioridad ligera desde el punto de vista de los síntomas negativos, no está claro si sus mejores cualidades se relacionaban con los síntomas negativos primarios o sólo con los secundarios. Los autores señalaron además que la superioridad más clara era para los fármacos atípicos con menos efectos secundarios extrapiramidales, un resultado sesgado por compararlo la mayoría de las veces con el haloperidol, que en muchos casos se daba en dosis altas ( $20 \mathrm{mg} /$ día).

Un meta-análisis más reciente realizado por Geddes y cols: [20] examinó 52 ensayos controlados que involucraron en conjunto a 12.649 pacientes. Los autores examinaron ensayos que comparaban amisulprida, clozapina, quetiapina, risperidona y sertindol frente a los fármacos convencionales (haloperidol o clorpromacina). Buscaron datos no posteriores al 1 de diciembre de 1998 en Medline, Embase, Psychlit y la biblioteca Cochrane. Con la ayuda de un grupo de expertos, localizaron también estudios inéditos y soli- citaron a las compañías farmacéuticas el acceso a sus datos no publicados (las compañías recibieron la solicitud dos veces en un intervalo de 1 mes). En particular, los autores examinaron variables de eficacia como las puntuaciones de la BPRS y la PANSS, así como las tasas de abandono y los efectos secundarios. También realizaron un análisis de regresión utilizando las dosis equivalentes de haloperidol como valor predictivo. Los resultados mostraron que la duración media de los estudios era 6,5 semanas y sólo cinco superaban el año. Observaron que muchos de los beneficios percibidos de los antipsicóticos atípicos se deben realmente a las dosis excesivas de haloperidol o clorpromacina utilizadas en los ensayos, y concluyeron que los "antipsicóticos atípicos tienen un efecto sobre los síntomas similar a los antipsicóticos convencionales en una dosis media de $<12 \mathrm{mg} /$ día de haloperidol." Los autores describieron entonces los resultados para cada medicación y encontraron que los pacientes que recibían antipsicóticos atípicos no tenían tasas de abandono más bajas o mejor respuesta que los pacientes que recibían la dosis óptima de los antipsicóticos convencionales, y llegaron a la conclusión general siguiente: "Los medicamentos convencionales deben ser el primer tratamiento". Este meta-análisis es un argumento suplementario para continuar el esfuerzo para demostrar que los ensayos psicofarmacológicos deben tener significado clínicamente.

Averiguar hasta qué punto un compuesto nuevo es superior clínicamente a un antipsicótico convencional requerirá hipótesis a priori adicionales basadas en marcos conceptuales que tengan significado clínicamente [33]. Es interesante advertir que, a pesar del curso a largo plazo de la esquizofrenia, la duración de la evaluación del tratamiento en el meta-análisis anterior era muy corta. No se puede negar que no hay en la actualidad datos convincentes sobre la materia en lo que se refiere a "largo plazo". Kapur y Remington comentaron hace poco que la mayoría de sus pacientes, que no pagan la medicación, prefieren los antipsicóticos atípicos a causa de la incidencia más baja de efectos secundarios [23]. Estos autores comunicaron que la combinación de los hallazgos de Geddes más su propia experiencia clínica "deja al profesional clínico en la cuerda floja entre la persuasividad de las afirmaciones de la comercialización, los resultados precisos pero algo miopes de los ensayos clínicos idealizados y las realidades complejas de la práctica clínica."

¿Cuál es la eficacia y la seguridad de los nuevos medicamentos antipsicóticos comparado con la efica- 
cia y la seguridad de la clozapina? El Grupo de Esquizofrenia de Cochrane ha realizado varios meta-análisis que estudiaban la eficacia de los antipsicóticos típicos y atípicos. En un intento por comparar los nuevos medicamentos antipsicóticos con la clozapina, los autores [36] identificaron ocho ensayos ciegos controlados distribuidos al azar que comparaban estas sustancias (795 pacientes) después de buscar en publicaciones en todas las lenguas de Biological Abstracts/BIOSIS (1980-1999), el Registro de Ensayos del Grupo de Esquizofrenia de Cochrane (1998), el registro CENTRAL de la Biblioteca Cochrane (número 4, 1999), EMBASE (1980-1998), MEDLINE (1966-1999), LILACS/CD-ROM (1998) y PsycLIT/PsycINFO (1974-1999). Además, se buscaron ensayos a partir de las actas de conferencias recientes y las listas de referencia de artículos incluidos. Se contactó con los autores de los ensayos recientes y los fabricantes de clozapina, iloperidona, olanzapina, quetiapina, remoxiprida, risperidona, sertindol, ziprasidona y zotepina. La duración de los ensayos era de 4 a 18 semanas. Los tamaños de muestra iban de 20 a 273. El estudio concluyó que los nuevos antipsicóticos y la clozapina no diferían cuando se utilizaba un índice clínico global que incluía la mejoría de los síntomas positivos y negativos. Pero este resultado se debía al pequeño número de estudios realizados $\mathrm{y}$, por tanto, se ha de interpretar con precaución. Por otra parte, encontraron que los efectos adversos diferían: la clozapina producía más fatiga, hipersalivación, náusea y mareo ortostático, mientras que los nuevos antipsicóticos atípicos, con la excepción de la olanzapina, producían más sintomas extrapiramidales. Los revisores concluyeron finalmente que: "La igualdad de la eficacia y la tolerabilidad de los nuevos medicamentos atípicos en comparación con la clozapina no está demostrada todavía". La revisión puso de relieve que se necesitan ensayos de poder suficiente, con duración más larga, que midan resultados clínicamente importantes, para evaluar la eficacia clínica, la tolerabilidad y la rentabilidad comparativas verdaderas de los nuevos medicamentos en relación con la clozapina. Un meta-análisis más reciente [5] basado en siete estudios que comparaban la clozapina con un antipsicótico típico reveló que los pacientes esquizofrénicos resistentes al tratamiento tienen una evolución más favorable con la clozapina. Sin embargo, el tamaño del efecto sobre las alteraciones psicopatológicas globales era sumamente variable, extendiéndose de 0,14 a 0,81 . Además, no hubo efectos de tratamiento significativos para la clozapina sobre los antipsicóticos convencio- nales en las puntuaciones para la subescala de síntomas positivos de la BPRS.

Las respuestas que el meta-análisis da a estas simples preguntas deberían producir en nosotros una gran humildad. Una cosa es cierta: si deseamos basar la psiquiatría en la $\mathrm{MBE}$, corremos el riesgo real de mirar más de cerca a lo que durante mucho tiempo se han considerado hechos.

Este aniversario nos concede el derecho a preguntar si los antipsicóticos funcionan, pero ¿lo estoy celebrando de manera ingenua? En primer lugar, ¿qué es la eficacia? ¿No deberíamos mencionar que significa una reducción del 20-40\% como mínimo de los síntomas positivos en los ensayos clínicos en una escala estandarizada? Un punto implícito en nuestra revisión clínica de los meta-análisis es saber si la reducción significativa de los síntomas positivos significa realmente que los neurolépticos funcionan así de bien. Desde luego, vale la pena debatir esto. Sabemos ahora, por ejemplo, y no lo sabíamos hace 50 años, que los síntomas positivos no correlacionaban con la evolución, pero los síntomas negativos y los cognitivos sí. Además, se supone que los nuevos antipsicóticos se dirigen a estos síntomas y los reducen mejor que los neurolépticos. Podría ser útil señalar que ninguno de estos agentes hace que la esquizofrenia remita, de modo que todavía queda un largo camino por recorrer en la eficacia. Debemos mencionar estas importantes deficiencias en el perfil de eficacia de los neurolépticos y los antipsicóticos atípicos.

En este momento, la responsabilidad y la honestidad aconsejan que aceptemos que todavía es necesario demostrar la eficacia de un gran número de nuestras herramientas terapéuticas en el tratamiento de los pacientes con esquizofrenia. Los psiquiatras deben sobre todo continuar dudando y siendo críticos. Debemos también militar en favor de la publicación de los resultados negativos, porque su inaccesibilidad modifica nuestro cuerpo de conocimientos como en todo, introduciendo típicamente un sesgo en favor de los nuevos fármacos. El profesional clínico, al enfrentarse a un paciente con esquizofrenia, que ha acudido esperando recibir un servicio, debe procesar implícita y explícitamente una gran cantidad de información, ponderarla críticamente y proponer luego el resultado de un compromiso. Después de todo, una de las funciones del profesional clínico es tranquilizar e informar al paciente. Si se basa en los meta-análisis, será honesto desde luego, pero hay una gran probabilidad de que no sea tranquilizador. Hans Lehman, que fue el primer psiquiatra que introdujo los neurolépticos en 
Norteamérica, en Montreal, Canadá, escribió en el artículo titulado "La historia de la psicofarmacología de la esquizofrenia" que el tratamiento eficaz de la esquizofrenia se logró sólo después de la introducción de los medicamentos antipsicóticos en la década de 1950 y está haciendo progresos todavía [28]. Celebrando el quincuagésimo aniversario de los neurolépticos y pensando en su eficacia, uno no puede citar a Umberto Eco (que citaba aparentemente a Boscoe Pertwee, un autor del siglo XVIII) en Kant y el ornitorrinco: "Solía ser indeciso, pero ahora no estoy tan seguro".

\section{BIBLIOGRAFÍA}

1 Abely P, Abdouchelli A, Fourment J, Schmitz B, Delteil P. Suite aux essais de re equilib ration ins ti nctivo-affective: essai de trai tement par le B.T.E.A. en comparaison avec le $4560 \mathrm{RP}$ et C.9295. Ann Med-Psychol 1952;110(2):262-7.

2 Arnold $\mathrm{OH}$, Hift S, Solms W. Uber die anwendung eines zentral vegetativen Himmungs-stoffes in der psychiatrischen Therapie. Wiener Med. Wochenschr 1952;102:965-9.

3 Azouri E. Traitement des états d'excitation et d'agitation par une phénothiazine d'action centrale élective. Jées Méd de Beyrouth. Expans. Scient 1952;2:293-5.

4 Carlsson A, Lindqvist M. Effect of chlorpromazine or haloperidol on formation of 3-methoxytyramine and norepinephrine in mouse brain. Psychiatr. Acta Pharmacol 1963;20:140-4.

5 Chakos M, Lieberman J, Hoffman E, Bradford D, Sheitman B. Effectiveness of second-generation antipsychotics in patients with treatment-resistant schizophrenia: A review and metaanalysis of randomised trials. Am J Psychiatr 2001;158: 518-26.

6 Chertok L. 130 years laten The story of the discovery of neuroleptics]. Ann Med-Psychol (Paris) 1982;140(9):971-6.

7 Delay J, Deniker P. 38 cas de psychoses traitées par la cure prolongée et continue de 4560 RP. C.R 50e Congrès des aliénistes et neurol. de Langue Fse 1952:503-13.

8 Delay J, Deniker P. (le 4560 RP utilisé seul en cure prolongee et continue) Le traitement des psychoses par une méthode neurolytique dérivée de l'hibernothérapie. C. R. $50 \mathrm{e}$ Congrès des aliénistes et neurol. de Langue Fse 1952:495-502.

9 Delay J, Deniker P. Reactions biologiques observées au cours du traitement par le Chlorte de diméthylaminopropyl-Nchlorophénothiazine. C.R. 50e Cong. des aliénistes et neurol. Langue Fse 1952:514-8.

10 Delay J, Deniker P, Harl JM. Traitement des états d'excitation et d'agitation par une méthode médicamenteuse derivee de l'hibernotherapie. Ann Med-Psychol 1952; 110(2) $: 267-73$.
11 Delay J, Deniker P, Harl JM. Utilisation en thérapeutique psychiatrique d'une phenothiazine, d'action centrale élective. Ann Med-Psychol 1952;110(2):112-7.

12. Delay J, Deniker P, Harl JM, Grasset A. Traitement d'états confusionnels par le Chlorp de diméthylaminopropyl-N-chloro-phénothiazine (4560 RP). Ann MedPsychol 1952; 110(2):398-403.

13 Deniker P. (brief history of the neuroleptics)From chlorpromazine to tardive dyskinesia. Psychiatr J Univ Ott 1989;14(1): 253-9.

14 Deniker P. [30 years of neuroleptic chemotherapy in psychiatry (evolution of the concept and practice)]. Bull Acad Natl Med 1982;166(1):113-21.

15 Deniker P. Impact of neuroleptic chemotherapies on schizophrenic psychoses. Am J. Psychiatr 1978;135(8):923-7.

16 Deniker P. Qui a inventé les neuroleptiques? Confront. Psychiatr 1975;13:7-17.

17 Deschamps A. Hibernation artificielle en psychiatrie. Presse Méd. 1952;60:944-5.

18 Deschamps A. Les ganglioplégiques en thérapeutique psychiatrique. Sem Med 1952:650-3.

19 Ey H, Berard E. Les nouvelles techniques de cures de sommeil dans la pratique psychiatrique. Evol Psychiatr 1952;4:661 -82.

20 Geddes J, Freemantle N, Harrison P, Beddington P. Atypical antipsychotics in the treatment of schizophrenia: Systematic overview and meta-regression analysis. Br Med J 2000;321: 1371-6:

21 Golse $\mathrm{J}$. Le traitement du délire aigu par l'hypernation. Ann Med-Psychol 1952;110(2):731-4.

22 Hamon J, Paraire J, Valluz J. Remarques sur l'action du 4560 RP sur l'agitation maniaque. Ann Med-Psychol 1952;110:331-5.

23 Kapur S, Remington G. Atypical antipsychotics. Br Med J 2000;321(7273):1360-1.

24 Kapur S, Seeman P. Does fast dissociation from the dopamine $d(2)$ receptor explain the action of atypical antipsychotics? A new hypothesis. Am J Psychiatr 2001 Mar;158(3):360-9.

25 Wadenberg ML, Soliman A, VanderSpek SC, Kapur S. Dopamine $\mathrm{d}(2)$ receptor occupancy is a common mechanism underlying animal models of antipsychotics and their clinical effects. Neuropsychopharmacology 2001;25(5):633-44.

26 Laborit $\mathrm{H}$, Huguenard P, Alluaume R. Un nouveau stabilisateur végétatif (le $4560 \mathrm{RP}$. Presse Med. 1952;60:206-8.

27 Leucht S, Pitschel-Walz, Abraham D, Kissling W. Efficacy and extrapyramidal side-effects of the new antipsychotics olanzapine, quetiapine, risperidone, and sertindole compared to conventional antipsychotics and placebo. A meta-analysis of randomized controlled trials. Schizophr Res 1999;35:51-68.

28 Lehmann HE, Ban TA. The history of the psychopharmacology of schizophrenia. Can J Psychiatr 1997 Mar;42(2):152-6. 
29 Loo H, Brochier T, Poirier MF, Olie JP. [40 years of neuroleptic drugs]. Ann Med-Psychol (Paris) 1993;151(3):2716.

30 Meltzer HY. Clinical studies on the mechanism of action of clozapine: the dopamine-serotonin hypothesis of schizophrenia. Psychopharmacology (Berlin) 1989;99(Suppl):S 18-27.

31 Meltzer HY. Biological studies in schizophrenia. Schizophr Bull 1987,13(1):77-111.

32 Pocidalo JJ, Cathala HP, Himbert J, Tardieu Mme. Action sur l'excitabilité sympathique du 4560 RP. C.R. Soc Biol 1952; 146:368-70.
33 Stip E. Novel antipsychotics: issues and controversies. Typicality of atypical antipsychotics. J Psychiatr Neurosci 2000;25:137-53.

34 Stip E, Liddle P, Soucy JP. Need for a new framework to understand the mechanism of all antipsychotics. $J$ Clin Psychiatr 2000;61(5):387-8.

35 Thornley B, Adams C. Content and quality of 2000 controlled trials in schizophrenia over 50 years. $\mathrm{Br}$ Med J 1998;317: 1181-4.

36 Tuunainen A, Wahlbeck K, Gilbody SM. Newer atypical antipsychotic medication versus clozapine for schizophrenia. Cochrane database Syst Rev 2000(3). 


\section{Desde el primer momento...}

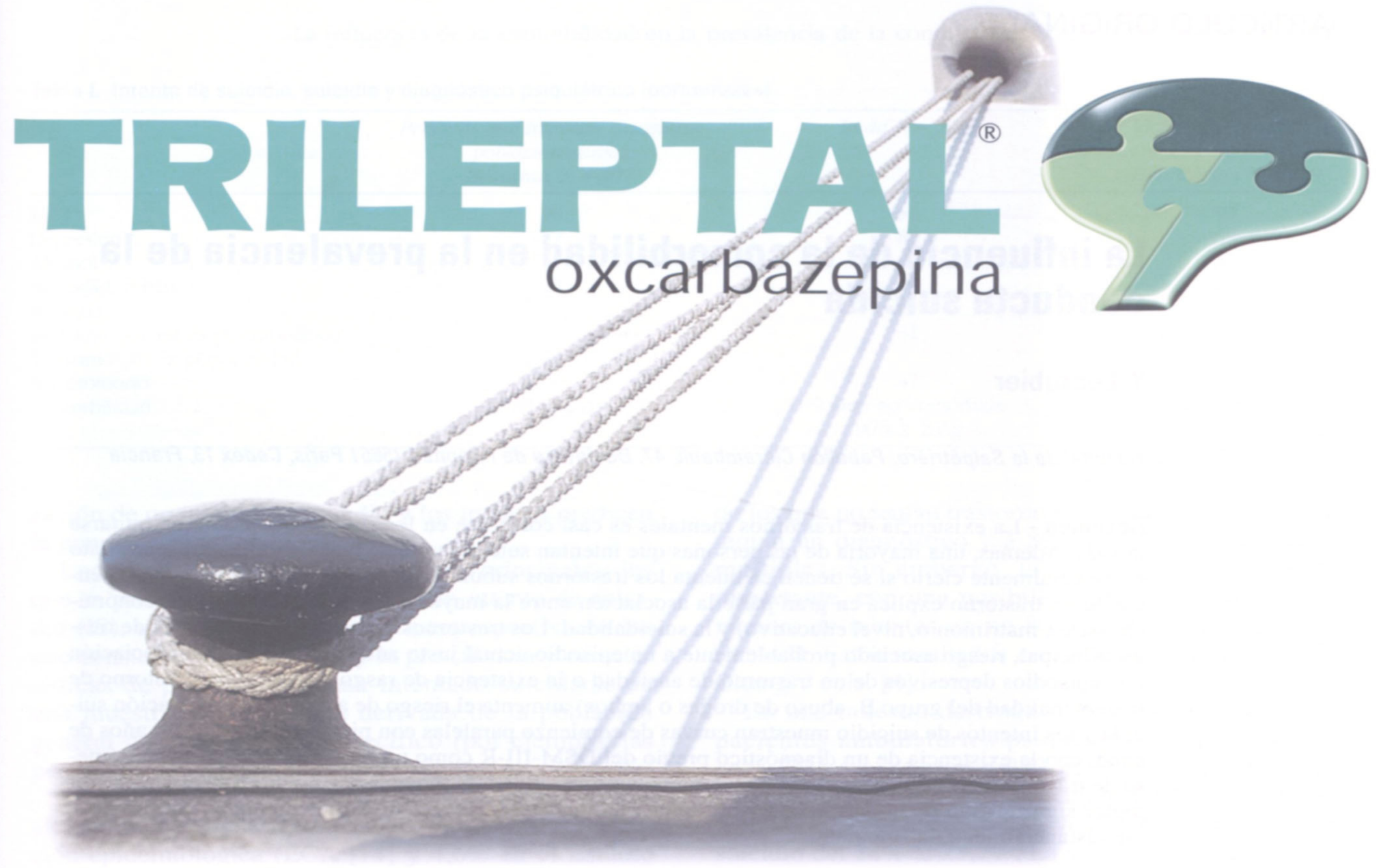

\section{$X$ EFICAZ, \\ $X$ SEGURO Y TOLERABLE X FÁCIL DE UTILIZAR,}

Trileptal $300 \mathrm{mg}$ Oxcarbazepina (DCI)

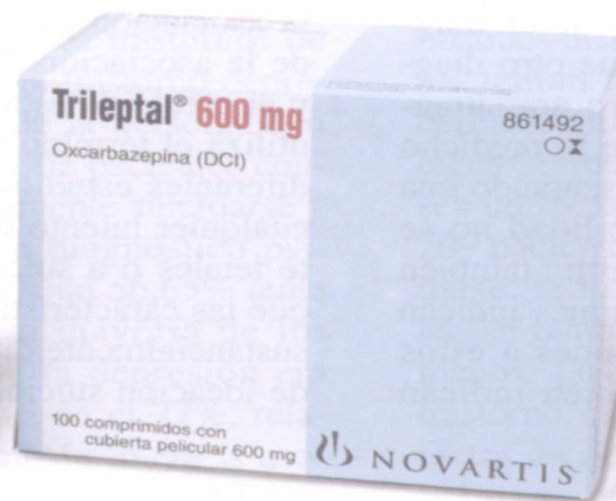

(1) NOVARTIS

Novartis Farmacéutica S.A

Gran Vía de les Corts Catalanes, 764

08013 Barcelona

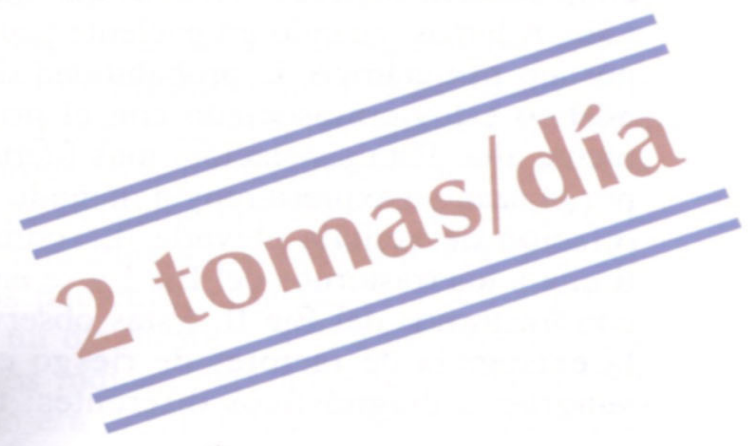




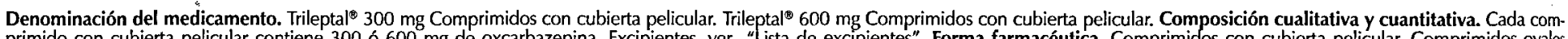

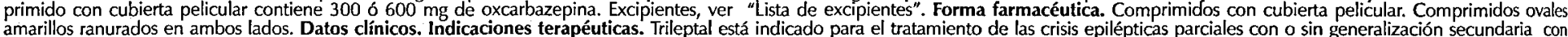

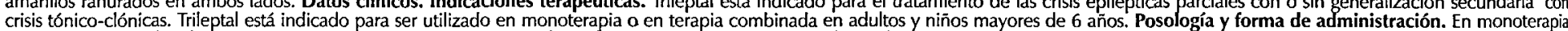

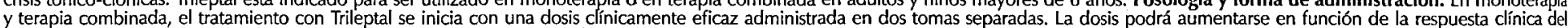

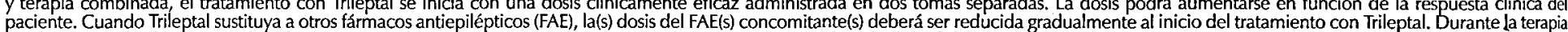

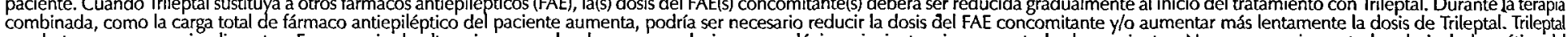

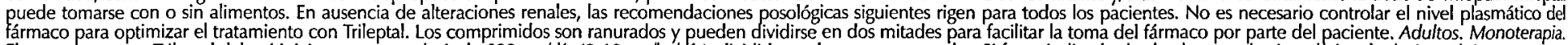

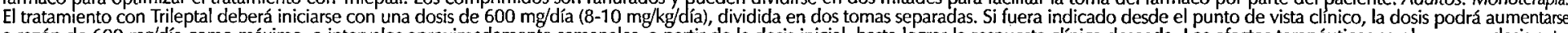

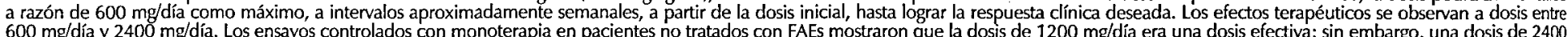

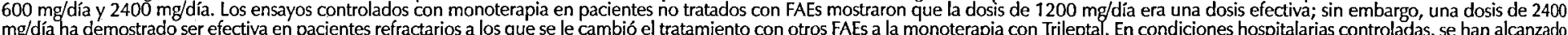

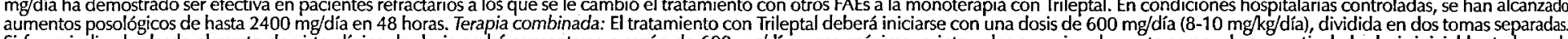

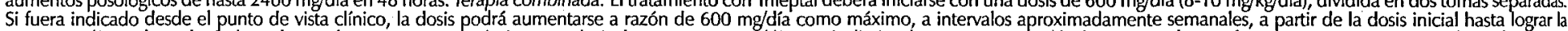

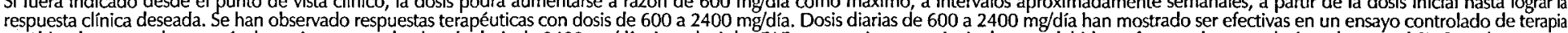

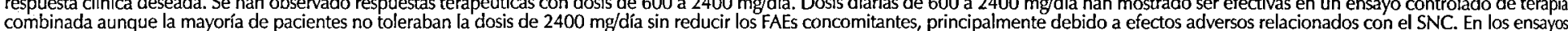

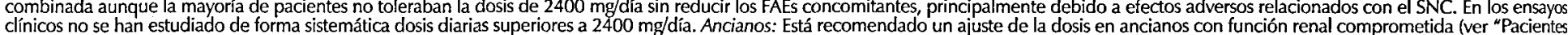

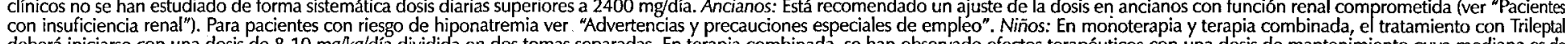

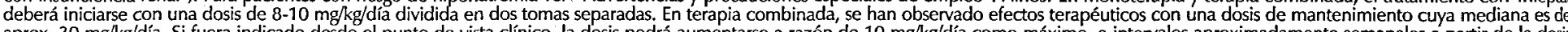

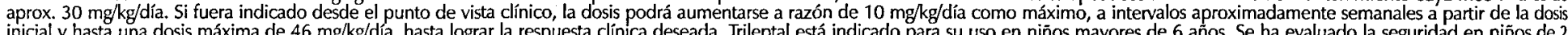

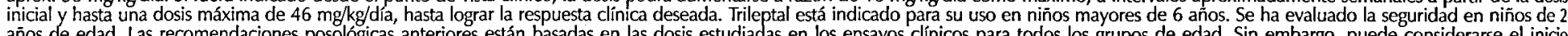

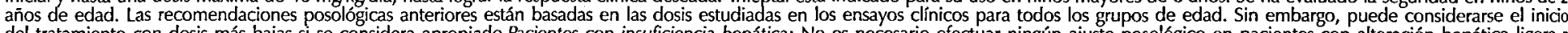

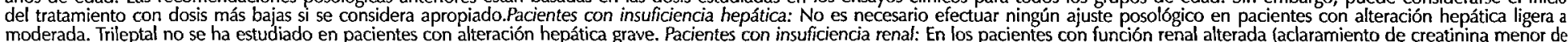

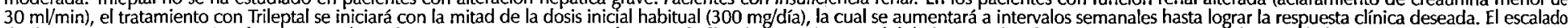

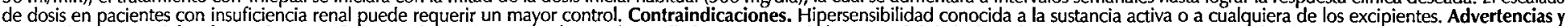

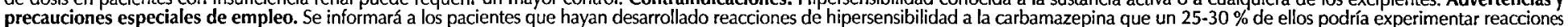

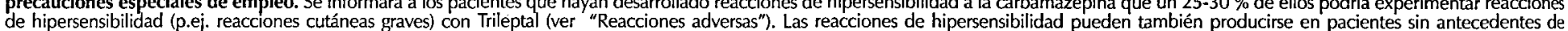

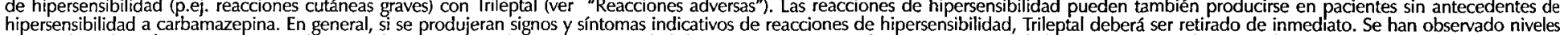

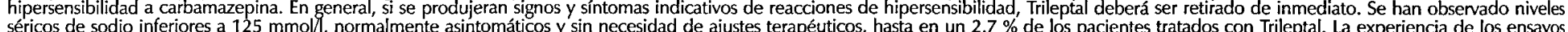

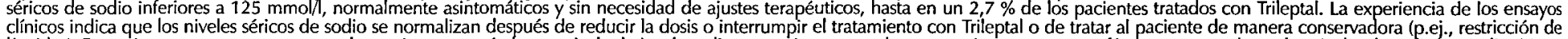

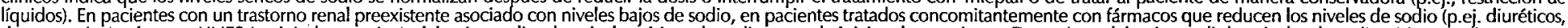

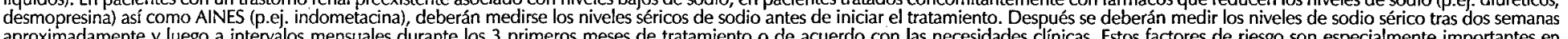

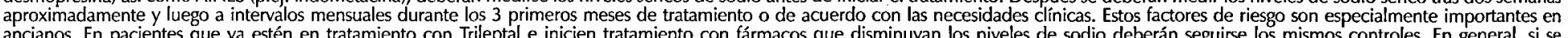

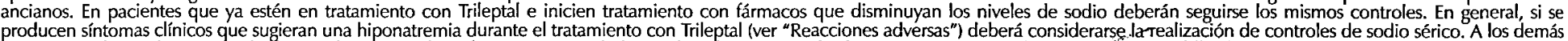

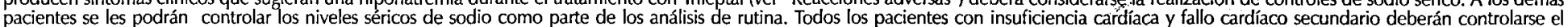

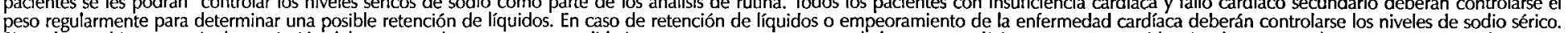

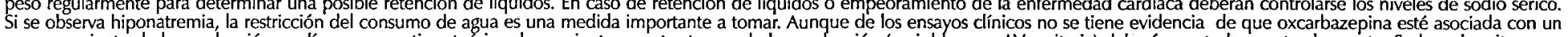

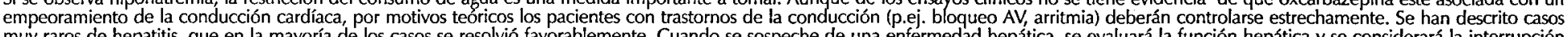

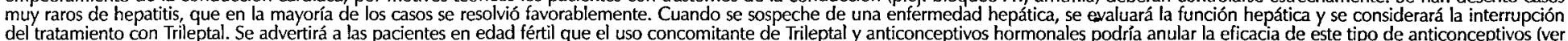

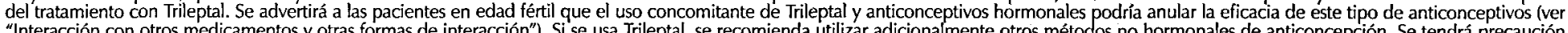

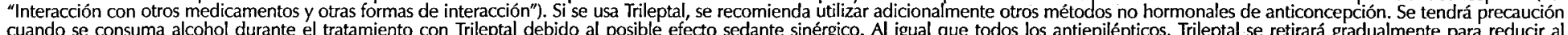

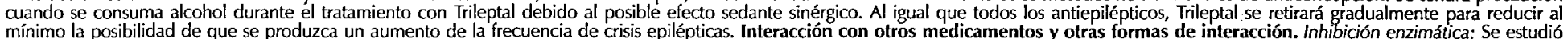

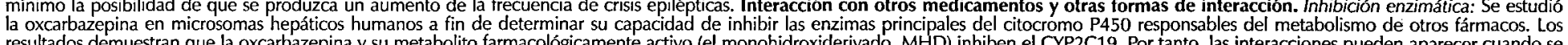

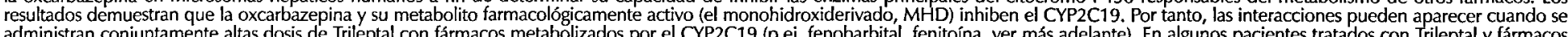

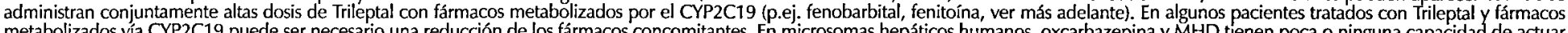

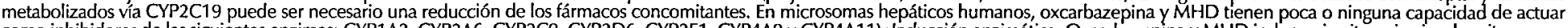

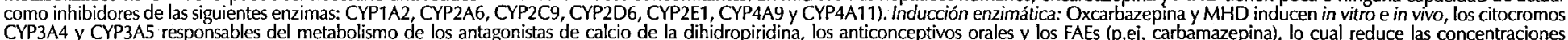

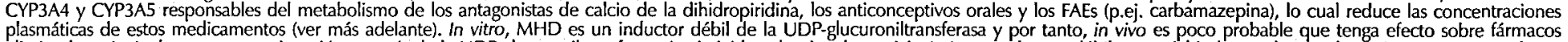

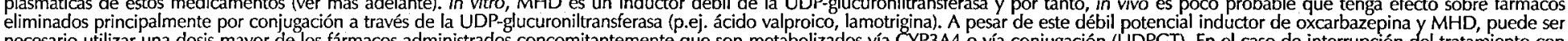

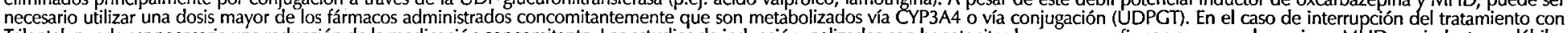

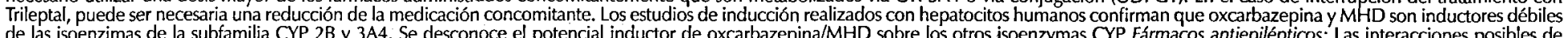

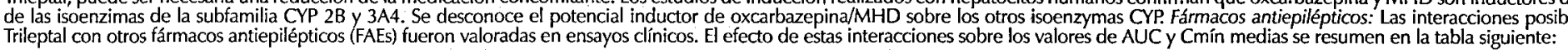

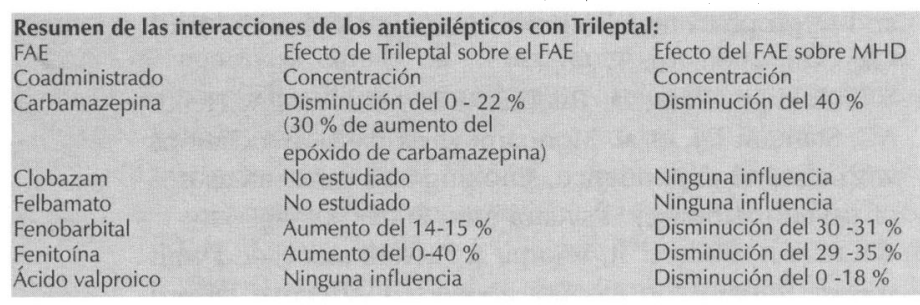

In vivo, los niveles plasmáticos de fenitoína aumentaron hasta en un $40 \%$ cuando se administró Trileptal a dosis superiores a $1200 \mathrm{mg} /$ día. Por tanto, cuando se usen dosis de Trileptal mayores de 1200 mg/día durante una terapia combinada podría ser necesario disminuir la dosis de fenitoína. El aumento de los niveles de fenobarbital, sin embargo, es pequeño (15\%) cuando se administra con Trileptal. Los inductores fuertes de las enzimas del citocromo $\mathrm{P450}$ (p.ej., carbamazepina, fenitoína y fenobarbital)
reducen los niveles plasmáticos de MHD (29-40\%). No se ha observado autoinducción con Trileptal. Anticonceptivos hormonales: Trileptal afecta a los dos componentes de un anticonceptivo oral - etinilestradiol (EE) y levonorgestrel (LNG). Los valores de AUC medios de EE y LNG disminuyeron entre un $48-52 \%$ y $32-52 \%$, respectivamente. No se han realizado estudios con otros anticonceptivos orales 0 implantes anticonceptivos. Por tanto, el uso concomitante de Trileptal y anticonceptivos hormonales podría anular la eficacia de estos últimos (ver "Advertencias y precauciones especiales de empleo"). felodipino disminuyeron en un $28 \%$. Sin embargo, los niveles plasmáticos permanecieron en el rango

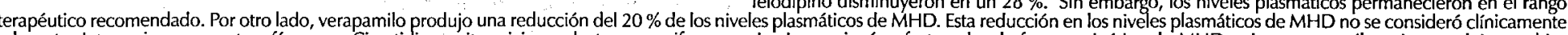

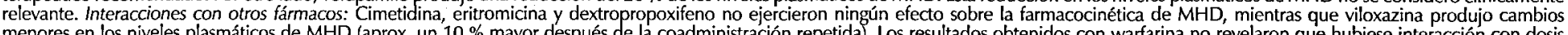

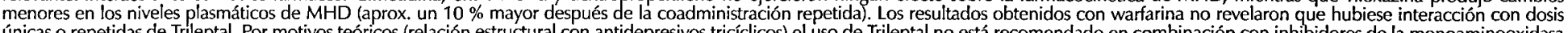

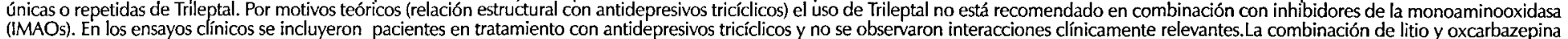

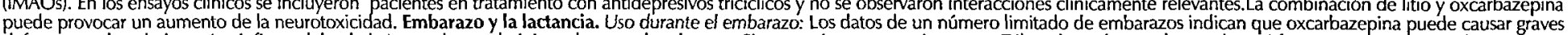

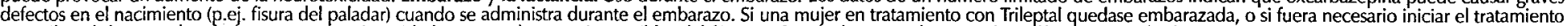

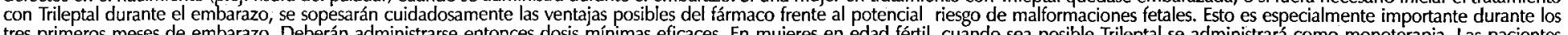

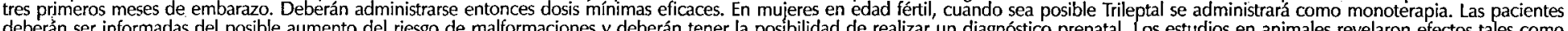

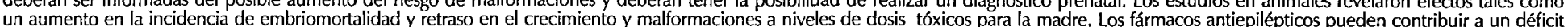

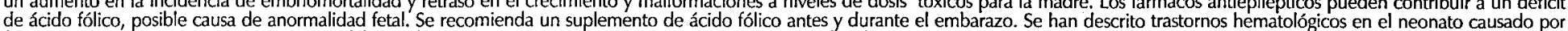

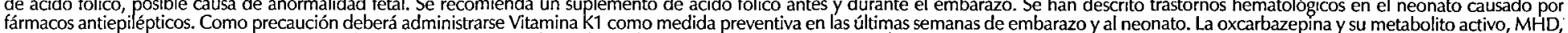

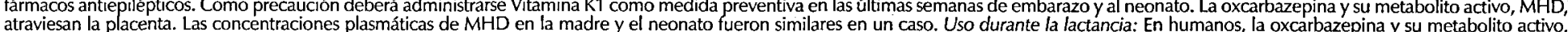

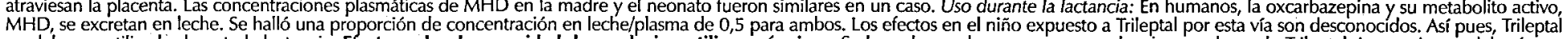

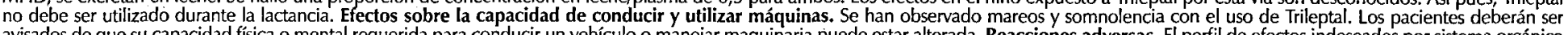

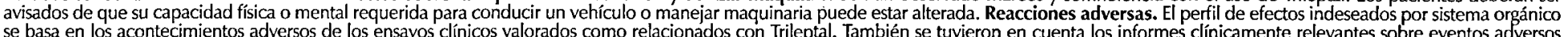

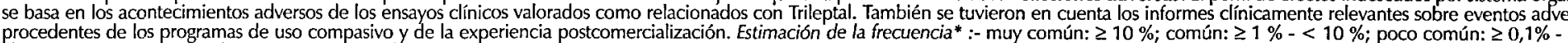

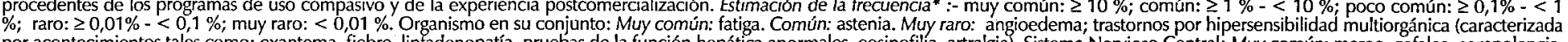

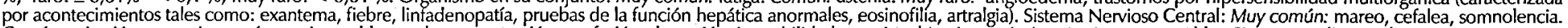

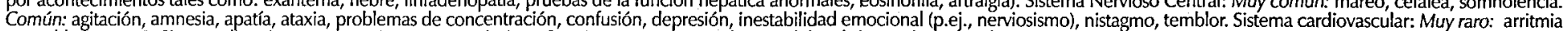

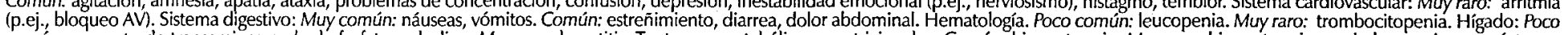

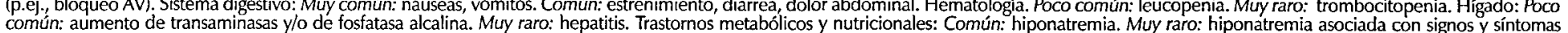

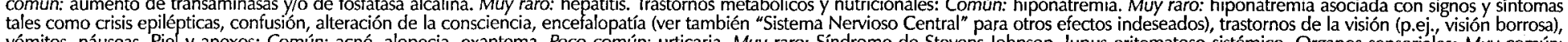

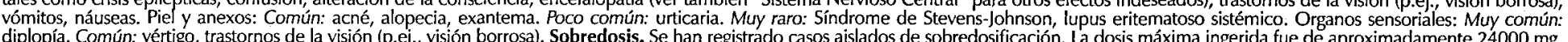

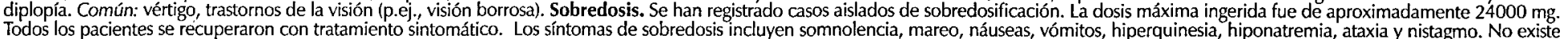

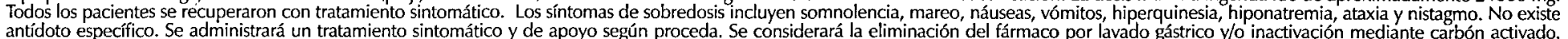

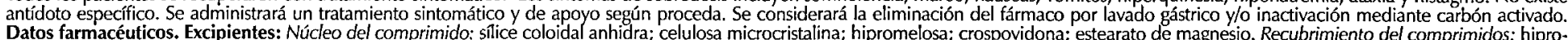

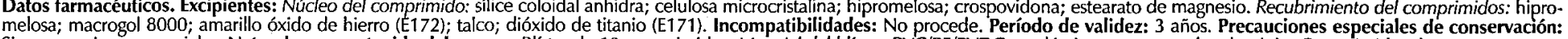

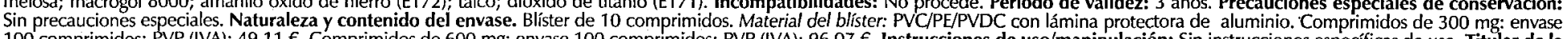

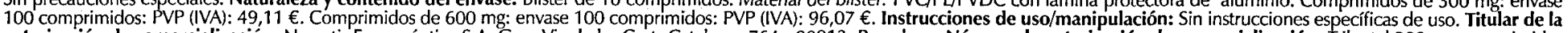

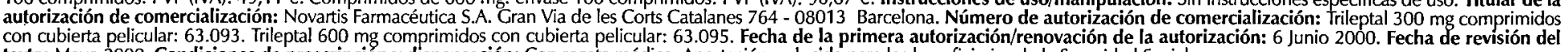
con cubierta pelicular: 63.093 . Trileptal $600 \mathrm{mg}$ comprimidos con cubierta pelicular: 63.095. Fecha de la primera autorización/renovación de la autoriza
texto: Mayo 2000. Condiciones de prescripción y dispensación: Con receta médica. Aportación reducida para los beneficiarios de la Seguridad Social.

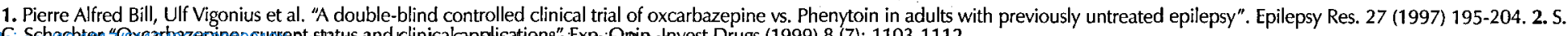

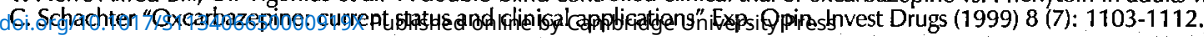

